\title{
ON CHUNG-TEICHER TYPE STRONG LAW FOR ARRAYS OF VECTOR-VALUED RANDOM VARIABLES
}

\author{
ANNA KUCZMASZEWSKA
}

Received 2 January 2003

\begin{abstract}
We study the equivalence between the weak and strong laws of large numbers for arrays of row-wise independent random elements with values in a Banach space $\mathscr{B}$. The conditions under which this equivalence holds are of the Chung or Chung-Teicher types. These conditions are expressed in terms of convergence of specific series and $o(1)$ requirements on specific weighted row-wise sums. Moreover, there are not any conditions assumed on the geometry of the underlying Banach space.
\end{abstract}

2000 Mathematics Subject Classification: 60F15, 60B12.

Let $(\Omega, \mathscr{F}, P)$ be a probability space and let $\mathscr{B}$ be a real separable Banach space with norm $\|\cdot\|$. A strongly measurable transformation from $\Omega$ to $\mathscr{B}$ is said to be a $\mathscr{B}$-valued random variable or a random element. If $E\|X\|<\infty$, then the expected value is defined by the Bochner integral.

Let $\left\{X_{n}, n \geq 1\right\}$ be a sequence of $\mathscr{B}$-valued random variables. Then $\left\{X_{n}, n \geq 1\right\}$ is said to obey the strong law of large numbers (SLLN) if there exist sequences of real numbers $\left\{a_{n}, n \geq 1\right\}$ and $\left\{b_{n}, n \geq 1\right\}$ such that

$$
\sum_{j=1}^{n} a_{j}\left(X_{j}-b_{j}\right) \longrightarrow 0 \quad \text { a.s., } n \longrightarrow \infty \text {. }
$$

Sufficient conditions for SLLN use very often the geometry of a Banach space, that is, they assume that $\mathscr{B}$ is a special-type space, for instance $\mathscr{B}$ is of Rademacher type $p$, $1<p \leq 2$.

The space $\mathscr{B}$ is of Rademacher type $p$ if there exists a positive constant $C$ such that

$$
E\left\|\sum_{n=1}^{\infty} \varepsilon_{n} x_{n}\right\|^{p} \leq C \sum_{n=1}^{\infty}\left\|x_{n}\right\|^{p}
$$

for each $\left(x_{1}, x_{2}, \ldots\right) \in C(B)$, where $\left\{\varepsilon_{n}, n \geq 1\right\}$ is a Bernoulli sequence, that is, $\varepsilon_{n}, n \geq 1$, are i.i.d. random variables and $P\left[\varepsilon_{n}=1\right]=P\left[\varepsilon_{n}=-1\right]=1 / 2, C(B)=\left\{\left(x_{1}, x_{2}, \ldots\right) \in B^{\infty}\right.$ : $\sum_{n=1}^{\infty} \varepsilon_{n} x_{n}$ converges in probability $\}, B^{\infty}=B \times B \times B \times \cdots$.

The sufficient conditions for SLLN for random elements taking value in a space of Rademacher type $p$ were presented by Woyczyński [15], Hoffmann-Jørgensen and Pisier [6], Kuczmaszewska and Szynal [8], and Adler et al. [1]. 
The type of Marcinkiewicz-Zygmunt SLLN provides that for $1 \leq \alpha<2$ and a sequence $\left\{X_{n}, n \geq 1\right\}$ of i.i.d. $\mathscr{B}$-valued random variables,

$$
\frac{1}{n^{1 / \alpha}} \sum_{i=1}^{n}\left(X_{i}-E X_{i}\right) \longrightarrow 0 \quad \text { a.s., } n \longrightarrow \infty,
$$

if and only if $E\left\|X_{1}\right\|<\infty$ and the Banach space $\mathscr{B}$ is of a Rademacher type $p$ for $\alpha<p \leq 2$ (cf. [15]).

The classical result of Hoffmann-Jørgensen and Pisier [6] proved that the assumption that a Banach space $\mathscr{B}$ is the space of Rademacher type $p, 1 \leq p \leq 2$, is equivalent to the fact that the condition

$$
\sum_{n=1}^{\infty} \frac{E\left\|X_{n}\right\|^{p}}{n^{p}}<\infty
$$

implies SLLN for a sequence of $\mathscr{B}$-valued independent random variables $\left\{X_{n}, n \geq 1\right\}$ with $E X_{n}=0, n \geq 1$.

In view of many statistical applications, it is important to consider the array-type SLLN.

Let $\left\{k_{n}, n \geq 1\right\}$ be a strictly increasing sequence of positive integers. An array of $\mathscr{B}$-valued random variables $\left\{X_{n i}, 1 \leq i \leq k_{n}, n \geq 1\right\}$ obeys the general array type of SLLN if

$$
\sum_{i=1}^{k_{n}} a_{n i}\left(X_{n i}-c_{n i}\right) \longrightarrow 0 \quad \text { a.s., } n \longrightarrow \infty
$$

where $\left\{a_{n i}, 1 \leq i \leq k_{n}, n \geq 1\right\}$ and $\left\{c_{n i}, 1 \leq i \leq k_{n}, n \geq 1\right\}$ are suitable arrays of constants (weights) and $\mathscr{B}$-valued elements, respectively, and 0 denotes the zero-element in $\mathscr{B}$.

$\mathrm{Hu}$ and Taylor [7] considered SLLN for arrays of row-wise independent random variables $\left\{X_{n i}, 1 \leq i \leq n, n \geq 1\right\}$.

Row-wise independence means that the random elements within each row are independent but no independence is assumed between rows.

In [3] Bozorgnia et al. obtained the Chung-type SLLN for arrays of row-wise independent random elements in a separable Banach space of Rademacher type $p, 1<p \leq 2$. They proved the following result.

THEOREM 1. Let $\left\{X_{n i}, 1 \leq i \leq n, n \geq 1\right\}$ be an array of row-wise independent random elements in a separable Banach space of Rademacher type $p, 1<p \leq 2$. Let $\varphi: \mathbb{R} \rightarrow \mathbb{R}$ be a positive, even, and continuous function such that

$$
\frac{\varphi(|x|)}{|x|^{r}} \succ, \frac{\varphi(|x|)}{|x|^{r+p-1}} \searrow \text { as }|x| \nearrow
$$

for some integer $r \geq 2$. 
Then the conditions

$$
\begin{gathered}
E X_{n i}=0, \quad 1 \leq i \leq n, n \geq 1, \\
\sum_{n=1}^{\infty} \sum_{i=1}^{n} \frac{E \varphi\left(\left\|X_{n i}\right\|\right)}{\varphi\left(a_{n}\right)}<\infty, \quad \sum_{n=1}^{\infty}\left[\sum_{i=1}^{n} E\left(\left\|\frac{X_{n i}}{a_{n}}\right\|^{p}\right)\right]^{p k}<\infty,
\end{gathered}
$$

for some positive integer $k$, imply

$$
\frac{1}{a_{n}} \sum_{i=1}^{n} X_{n i} \longrightarrow 0 \quad \text { a.s., } n \longrightarrow \infty,
$$

where $\left\{a_{n}, n \geq 1\right\}$ is a sequence of positive increasing real numbers such that

$$
\lim _{n \rightarrow \infty} a_{n}=\infty .
$$

This theorem generalizes $\mathrm{Hu}$ and Taylor's result (cf. [7]) on the case of $\mathscr{B}$-valued random variables $\left\{X_{n i}, 1 \leq i \leq n, n \geq 1\right\}$ taking value in a Banach space of Rademacher type $p$. Moreover, the assumptions of the function $\varphi$ have some relationships with the geometric condition Rademacher type $p$ of the Banach space.

Some results which consider the problem of equivalence between weak law of large numbers (WLLN) and SLLN for a sequence $\left\{X_{n}, n \geq 1\right\}$ of independent $\mathscr{B}$-valued random variables can be found in Kuelbs and Zinn [10], de Acosta [4], Etemadi [5], Mikosch and Norvaiša [11, 12], Wang et al. [14], and Kuczmaszewska and Szynal [9].

Now, we recall some definitions and a lemma which will be used in the paper.

Definition 2. A double array $\left\{a_{n i}, i \geq 1, n \geq 1\right\}$ of real numbers is said to be a Toeplitz array if $\lim _{n \rightarrow \infty} a_{n i}=0$ for each $i \geq 1$ and $\sum_{i=1}^{\infty}\left|a_{n i}\right| \leq C$ for all $n \geq 1$, where $C>0$.

In further consideration, we need an extension of the concept of stochastic domination by a random variable to an array of $\mathscr{B}$-valued random variables.

An array $\left\{X_{n i}, i \geq 1, n \geq 1\right\}$ of $\mathscr{B}$-valued random variables is stochastically dominated by the random element $X$ if there exists a constant $D>0$ such that

$$
P\left[\left\|X_{n i}\right\|>x\right] \leq D P[D\|X\|>x]
$$

for all $x \geq 0, i \geq 1$, and $n \geq 1$.

We also need some inequalities which will be very important in our consideration. The following lemma presents one of them.

LEMMA 3 (cf. Yurinskii [16]). Let $X_{1}, X_{2}, \ldots, X_{n}$ be independent $\mathscr{B}$-valued random variables with $E\left\|X_{i}\right\|<\infty, i=1,2, \ldots, n$. Let $\mathscr{F}$ be a $\sigma$-field generated by $\left(X_{1}, X_{2}, \ldots, X_{k}\right)$, $k=1,2, \ldots, n$, and let $\mathscr{F}_{0}=\{\Omega, \varnothing\}$. Then for $1 \leq k \leq n$ and $S_{n}=\sum_{i=1}^{n} X_{i}$,

$$
\left|E\left(\| S_{n}|| \mid \mathscr{F}_{k}\right)-E\left(|| S_{n}|| \mid \mathscr{F}_{k-1}\right)\right| \leq\left\|X_{k}\right\|+E\left\|X_{k}\right\| .
$$

THEOREM 4. Let $\left\{X_{n i}, 1 \leq i \leq k_{n}, n \geq 1\right\}$ be an array of row-wise independent $\mathscr{B}$ valued random variables with $E X_{n i}=0$ for all $1 \leq i \leq k_{n}, n \geq 1$, and for some increasing 
sequence $\left\{k_{n}, n \geq 1\right\}$ of positive integers. Let $\varphi_{n i}: \mathbb{R} \rightarrow \mathbb{R}_{+}$and $\psi_{n i}: \mathbb{R} \rightarrow \mathbb{R}_{+}$be positive, even, and continuous functions, which for constants $\alpha_{n i} \geq 1,0<\beta_{n i} \leq 2, K_{n i}>0$, and $M_{n i}>0,1 \leq i \leq k_{n}, n \geq 1$, satisfy the following conditions:

$$
\begin{aligned}
& \left|x_{1}\right| \leq\left|x_{2}\right| \Longrightarrow \frac{\varphi_{n i}\left(\left|x_{1}\right|\right)}{\left|x_{1}\right|^{\alpha_{n i}}} \leq K_{n i} \frac{\varphi_{n i}\left(\left|x_{2}\right|\right)}{\left|x_{2}\right|^{\alpha_{n i}}}, \\
& \left|x_{1}\right| \leq\left|x_{2}\right| \Longrightarrow \frac{\left|x_{1}\right|^{\beta_{n i}}}{\psi_{n i}\left(\left|x_{1}\right|\right)} \leq M_{n i} \frac{\left|x_{2}\right|^{\beta_{n i}}}{\psi_{n i}\left(\left|x_{2}\right|\right)} .
\end{aligned}
$$

Suppose that for some array $\left\{a_{n i},\left(1 \leq i \leq k_{n}, n \geq 1\right)\right\}$ of nonzero reals and $k \geq 1 / 2$,

$$
\begin{gathered}
\sum_{n=1}^{\infty} E\left(\sum_{i=1}^{k_{n}} M_{n i} \frac{\psi_{n i}\left(\left\|X_{n i}\right\|\right)}{\psi_{n i}\left(a_{n i}^{-1}\right)}\right)^{k}<\infty, \\
\sum_{n=1}^{\infty} \sum_{i=1}^{k_{n}} P\left[\left\|X_{n i}\right\| \geq c_{n i}\right]<\infty,
\end{gathered}
$$

for some array $\left\{c_{n i}, 1 \leq i \leq k_{n}, n \geq 1\right\}$ of positive numbers such that

$$
\sum_{n=1}^{\infty} \sum_{i=1}^{n} K_{n i}^{2} \cdot \varphi_{n i}\left(c_{n i}\right) \frac{E \varphi_{n i}\left(\left\|X_{n i}\right\|\right)}{\varphi_{n i}^{2}\left(a_{n i}^{-1}\right)}<\infty .
$$

Then

$$
\sum_{i=1}^{k_{n}} a_{n i} X_{n i} \stackrel{P}{\longrightarrow} 0, \quad n \longrightarrow \infty,
$$

if and only if

$$
\sum_{i=1}^{k_{n}} a_{n i} X_{n i} \longrightarrow 0 \quad \text { a.s., } n \longrightarrow \infty .
$$

Proof. Let $X_{n i}^{\prime}=X_{n i} I\left[\left\|X_{n i}\right\| \leq\left|a_{n i}^{-1}\right|\right]$ and $X_{n i}^{*}=X_{n i}^{\prime}-E X_{n i}^{\prime}$.

Now we introduce the following notation:

$$
S_{n}=\sum_{i=1}^{k_{n}} a_{n i} X_{n i}, \quad S_{n}^{\prime}=\sum_{i=1}^{k_{n}} a_{n i} X_{n i}^{\prime} .
$$

Note that using this notation, condition (12) on the Borel functions $\varphi_{n i}$, and assumptions (15) and (16), we have

$$
\begin{aligned}
\sum_{n=1}^{\infty} P\left[S_{n} \neq S_{n}^{\prime}\right] & =\sum_{n=1}^{\infty} P\left[\left\|\sum_{i=1}^{k_{n}} a_{n i} X_{n i} I\left[\left\|X_{n i}\right\|>\left|a_{n i}^{-1}\right|\right]\right\|>\varepsilon\right] \\
& \leq \sum_{n=1}^{\infty} \sum_{i=1}^{k_{n}} P\left[\left\|X_{n i}\right\| I\left[\left\|X_{n i}\right\|>\left|a_{n i}^{-1}\right|\right] \neq 0\right] \\
& =\sum_{n=1}^{\infty} \sum_{i=1}^{k_{n}} P\left[\left\|X_{n i}\right\|>\left|a_{n i}^{-1}\right|\right]
\end{aligned}
$$


ON CHUNG-TEICHER TYPE STRONG LAW ...

$$
\begin{aligned}
\leq & \sum_{n=1}^{\infty} \sum_{i=1}^{k_{n}} E I\left[\left\|X_{n i}\right\|>\left|a_{n i}^{-1}\right|\right] \cdot I\left[\left\|X_{n i}\right\| \geq c_{n i}\right] \\
& +\sum_{n=1}^{\infty} \sum_{i=1}^{k_{n}} E I\left[\left\|X_{n i}\right\|>\left|a_{n i}^{-1}\right|\right] \cdot I\left[\left\|X_{n i}\right\|<c_{n i}\right] \\
\leq & \sum_{n=1}^{\infty} \sum_{i=1}^{k_{n}} P\left[\left\|X_{n i}\right\| \geq c_{n i}\right] \\
& +\sum_{n=1}^{\infty} \sum_{i=1}^{k_{n}} E\left\{\left(\frac{\left\|X_{n i}\right\|^{\alpha_{n i}}}{\left(\left|a_{n i}^{-1}\right|\right)^{\alpha_{n i}}}\right)^{2} I\left[\left\|X_{n i}\right\|>\left|a_{n i}^{-1}\right|\right] \cdot I\left[\left\|X_{n i}\right\|<c_{n i}\right]\right\} \\
\leq & \sum_{n=1}^{\infty} \sum_{i=1}^{k_{n}} P\left[\left\|X_{n i}\right\| \geq c_{n i}\right]+\sum_{n=1}^{\infty} \sum_{i=1}^{k_{n}} K_{n i}^{2} \cdot \varphi_{n i}\left(c_{n i}\right) \frac{E \varphi_{n i}\left(\| X_{n i}||\right)}{\varphi_{n i}^{2}\left(a_{n i}^{-1}\right)}<\infty .
\end{aligned}
$$

Thus the two sequences $\left\{S_{n}, n \geq 1\right\}$ and $\left\{S_{n}^{\prime}, n \geq 1\right\}$ are equivalent.

Now we must prove that

$$
E\left\|S_{n}^{\prime}\right\| \longrightarrow 0, \quad n \longrightarrow \infty
$$

First we will show that

$$
\left\|S_{n}^{\prime}\right\|-E\left\|S_{n}^{\prime}\right\| \stackrel{P}{\longrightarrow} 0, \quad n \rightarrow \infty
$$

Using the Markov inequality, the Marcinkiewicz-Zygmunt inequality in its Banach space version (cf. de Acosta [4] or Berger [2]), and assumptions (12) and (14), for any $\varepsilon>0$, we get

$$
\begin{aligned}
P\left[\left\|S_{n}^{\prime}\right\|-E\left\|S_{n}^{\prime}\right\| \mid>\varepsilon\right] \leq & \varepsilon^{-2 k} E\left|\left\|S_{n}^{\prime}\right\|-E\left\|S_{n}^{\prime}\right\|\right|^{2 k} \\
& \leq \varepsilon^{-2 k} A_{k} E\left(\sum_{i=1}^{k_{n}}\left\|a_{n i} X_{n i}^{\prime}\right\|^{2}\right)^{k}=\varepsilon^{-2 k} A_{k} E\left(\sum_{i=1}^{k_{n}} \frac{\left\|X_{n i}^{\prime}\right\|^{2}}{\left(a_{n i}^{-1}\right)^{2}}\right)^{k} \\
& =\varepsilon^{-2 k} A_{k} E\left(\sum_{i=1}^{k_{n}} \frac{\left\|X_{n i}^{\prime}\right\|^{\beta_{n i}}}{\left(\left|a_{n i}^{-1}\right|\right)^{\beta_{n i}}} \cdot \frac{\left\|X_{n i}^{\prime}\right\|^{2-\beta_{n i}}}{\left(\left|a_{n i}^{-1}\right|\right)^{2-\beta_{n i}}}\right)^{k} \\
& \leq \varepsilon^{-2 k} A_{k} E\left(\sum_{i=1}^{k_{n}} M_{n i} \frac{\psi_{n i}\left(\left\|X_{n i}^{\prime}\right\|\right)}{\psi_{n i}\left(a_{n i}^{-1}\right)}\right)^{k} \\
& \times \varepsilon^{-2 k} A_{k} E\left(\sum_{i=1}^{k_{n}} M_{n i} \frac{\psi_{n i}\left(\left\|X_{n i}\right\|\right)}{\psi_{n i}\left(a_{n i}^{-1}\right)}\right)^{k}=o(1) .
\end{aligned}
$$

Thus we conclude that (22) holds and, together with (17) and the equivalence between $\left\{S_{n}, n \geq 1\right\}$ and $\left\{S_{n}^{\prime}, n \geq 1\right\}$, gives (21). 
Now, we will show that $\left\|S_{n}^{\prime}\right\| \rightarrow 0$ a.s., as $n \rightarrow \infty$. By (21) it is enough to prove that

$$
\left\|S_{n}^{\prime}\right\|-E\left\|S_{n}^{\prime}\right\| \longrightarrow 0 \quad \text { a.s., } n \rightarrow \infty
$$

As before, using the Markov inequality, the Marcinkiewicz-Zygmunt inequality, condition (13), and assumption (14), we have

$$
\begin{aligned}
\sum_{n=1}^{\infty} P\left[\left\|S_{n}^{\prime}\right\|-E\left\|S_{n}^{\prime}\right\| \mid>\varepsilon\right] & \leq \varepsilon^{-2 k} \sum_{n=1}^{\infty} E\left|\left\|S_{n}^{\prime}\right\|-E\left\|S_{n}^{\prime}\right\|\right|^{2 k} \\
& \leq \varepsilon^{-2 k} A_{k} \sum_{n=1}^{\infty} E\left(\sum_{i=1}^{k_{n}} \frac{\left\|X_{n i}^{\prime}\right\|^{2}}{\left(a_{n i}^{-1}\right)^{2}}\right)^{k} \\
& =\varepsilon^{-2 k} A_{k} \sum_{n=1}^{\infty} E\left(\sum_{i=1}^{k_{n}} \frac{\left\|X_{n i}^{\prime}\right\|^{\beta_{n i}}}{\left(\left|a_{n i}^{-1}\right|\right)^{\beta_{n i}}} \cdot \frac{\left\|X_{n i}^{\prime}\right\|^{2-\beta_{n i}}}{\left(\left|a_{n i}^{-1}\right|\right)^{2-\beta_{n i}}}\right)^{k} \\
& \leq \varepsilon^{-2 k} A_{k} \sum_{n=1}^{\infty} E\left(\sum_{i=1}^{k_{n}} M_{n i} \frac{\psi_{n i}\left(\left\|X_{n i}\right\|\right)}{\psi_{n i}\left(a_{n i}^{-1}\right)}\right)^{k}<\infty
\end{aligned}
$$

Hence, by the Borel-Cantelli lemma, we obtain (24), which, by the equivalence between $\left\{S_{n}, n \geq 1\right\}$ and $\left\{S_{n}^{\prime}, n \geq 1\right\}$, completes the proof.

Note that if we put in Theorem $4 \varphi_{n i}=\varphi$ and $\psi_{n i}=\psi$, where $\varphi: \mathbb{R} \rightarrow \mathbb{R}_{+}, \psi: \mathbb{R} \rightarrow \mathbb{R}_{+}$ are positive, even, and continuous functions such that

$$
\frac{\varphi(|x|)}{|x|^{\alpha}} \nearrow, \frac{\psi(|x|)}{|x|^{\beta}} \searrow \text { as }|x| \nearrow
$$

for some $\alpha \geq 1$ and $0<\beta \leq 2$, we get the following result.

COROLlary 5. Let $\left\{X_{n i}, 1 \leq i \leq k_{n}, n \geq 1\right\}$ be an array of row-wise independent $\mathscr{B}$ valued random variables with $E X_{n i}=0$ for all $1 \leq i \leq k_{n}, n \geq 1$, and for any increasing sequence $\left\{k_{n}, n \geq 1\right\}$ of positive integers. Let $\varphi: \mathbb{R} \rightarrow \mathbb{R}_{+}$and $\psi: \mathbb{R} \rightarrow \mathbb{R}_{+}$be positive, even, and continuous functions satisfying (26) for some $\alpha \geq 1$ and $0<\beta \leq 2$.

Suppose that for some array $\left\{a_{n i}, 1 \leq i \leq k_{n}, n \geq 1\right\}$ of nonzero reals and $k \geq 1 / 2$,

$$
\sum_{n=1}^{\infty} E\left(\sum_{i=1}^{k_{n}} \frac{\psi\left(\left\|X_{n i}\right\|\right)}{\psi\left(a_{n i}^{-1}\right)}\right)^{k}<\infty, \quad \sum_{n=1}^{\infty} \sum_{i=1}^{k_{n}} P\left[\left\|X_{n i}\right\| \geq c_{n i}\right]<\infty,
$$

for some array $\left\{c_{n i},(1 \leq i \leq 1, n \geq 1)\right\}$ of positive numbers such that

$$
\sum_{n=1}^{\infty} \sum_{i=1}^{k_{n}} \varphi\left(c_{n i}\right) \frac{E \varphi\left(\left\|X_{n i}\right\|\right)}{\varphi^{2}\left(a_{n i}^{-1}\right)}<\infty \text {. }
$$

Then (17) is equivalent to (18).

Putting $\psi(x)=|x|^{p}, 1<p \leq \beta \leq 2$, and $c_{n i}=\left|a_{n i}^{-1}\right|$, we obtain the following result for a separable Banach space of Rademacher type $p$. 
COROLlary 6. Let $\left\{X_{n i}, 1 \leq i \leq k_{n}, n \geq 1\right\}$ be an array of row-wise independent $\mathscr{B}$ valued random variables in a separable Banach space of Rademacher type $p, 1<p \leq \beta \leq$ 2 , with $E X_{n i}=0$ for all $1 \leq i \leq k_{n}, n \geq 1$, and for some increasing sequence $\left\{k_{n}, n \geq 1\right\}$ of positive integers. Let $\varphi: \mathbb{R} \rightarrow \mathbb{R}$ be a positive, even, and continuous function such that

$$
\frac{\varphi(|x|)}{|x|^{\alpha}}, \quad \text { as }|x| \nearrow
$$

for some $\alpha \geq 1$.

Then, for some array $\left\{a_{n i},\left(1 \leq i \leq k_{n}, n \geq 1\right)\right\}$ of nonzero reals and some integer $k \geq 1$, the conditions

$$
\begin{gathered}
\sum_{n=1}^{\infty}\left[\sum_{i=1}^{k_{n}} \frac{E\left(\left\|X_{n i}\right\|^{p}\right)}{\left|a_{n i}\right|^{-p}}\right]^{k}<\infty, \\
\sum_{n=1}^{\infty} \sum_{i=1}^{k_{n}} \frac{E \varphi\left(\| X_{n i}||\right)}{\varphi\left(a_{n i}^{-1}\right)}<\infty
\end{gathered}
$$

imply (18).

Proof. Putting $M_{n i}=K_{n i}=1$ and using (20), we see that it is enough to show that Theorem 4 holds for $\left\{X_{n i}, 1 \leq i \leq k_{n}, n \geq 1\right\}$ and $k \geq 2$.

Indeed, we have

$$
\begin{aligned}
& \sum_{n=1}^{\infty} E\left(\sum_{i=1}^{k_{n}} \frac{\|\left. X_{n i}^{\prime}\right|^{p}}{\left|a_{n i}^{-1}\right|^{p}}\right)^{k} \\
& \quad \leq \sum_{n=1}^{\infty} \sum^{*}\left(\begin{array}{c}
k \\
s_{1}, \ldots, s_{k_{n}}
\end{array}\right) E\left(\frac{\|\left. X_{n 1}^{\prime}\right|^{p}}{\left|a_{n 1}^{-1}\right|^{p}}\right)^{s_{1}} E\left(\frac{\|\left. X_{n 2}^{\prime}\right|^{p}}{\left|a_{n 2}^{-1}\right|^{p}}\right)^{s_{2}} \cdots E\left(\frac{\left\|X_{n k_{n}}^{\prime}\right\|^{p}}{\left|a_{n k_{n}}^{-1}\right|^{p}}\right)^{s_{k_{n}}},
\end{aligned}
$$

where the sum $\sum *\left(\begin{array}{c}k \\ s_{1}, \ldots, s_{k_{n}}\end{array}\right)$ is over all choices of $\left\{s_{1}, s_{2}, \ldots, s_{k_{n}}\right\}, s_{i} \in\{0,1,2, \ldots, k\}$, such that $\sum_{i=1}^{k_{n}} s_{i}=k$. Choose $n$ sufficiently large so that $k_{n}>k$. Let $m=m\left(s_{1}, s_{2}, \ldots, s_{k_{n}}\right)$ be a number of $s_{i} \neq 0$. We see that $m$ takes all the values from the set $\{1,2, \ldots, k\}$. Changing the order in our sum, we can express the right-hand side of (32) in the following form:

$$
\begin{aligned}
& \sum_{n=1}^{\infty} \sum_{m=1}^{k} \sum_{\substack{1 \leq i_{j} \leq k_{n}, j=1,2, \ldots, m \\
i_{j} \neq i_{k}, \forall k \neq j}} *\left(\begin{array}{c}
k \\
s_{i_{1}}, \ldots, s_{i_{m}}
\end{array}\right) E\left(\frac{\|\left. X_{n i_{1}}^{\prime}\right|^{p}}{\left|a_{n i_{1}}^{-1}\right|^{p}}\right)^{s_{i_{1}}} E\left(\frac{\|\left. X_{n i_{2}}^{\prime}\right|^{p}}{\left|a_{n i_{2}}^{-1}\right|^{p}}\right)^{s_{i_{2}}} \cdots E\left(\frac{\|\left. X_{n i_{m}}^{\prime}\right|^{p}}{\left|a_{n i_{m}}^{-1}\right|^{p}}\right)^{s_{i_{m}}} \\
& \quad \leq \sum_{n=1}^{\infty}\left\{\sum_{\substack{1 \leq i_{1}<i_{2}<\cdots<i_{k} \leq\left. k_{n} \\
\sum_{n i_{1}}\right|^{p}}} E\left(\frac{\|\left. X_{n i_{1}}^{\prime}\right|^{p}}{\left|a_{1}^{-1}\right|^{p}}\right) E\left(\frac{\|\left. X_{n i_{2}}^{\prime}\right|^{p}}{\left|a_{n i_{2}}^{-1}\right|^{p}}\right) \cdots E\left(\frac{\|\left. X_{n i_{k}}^{\prime}\right|^{p}}{\left|a_{n i_{k}}^{-1}\right|^{p}}\right)\right.
\end{aligned}
$$




$$
\begin{gathered}
+\left(\sum_{\substack { m=1 \\
\begin{subarray}{c}{1 \leq i_{j} \leq k_{n}, j=1,2, \ldots, m \\
i_{j} \neq i_{k} \forall k \neq j{ m = 1 \\
\begin{subarray} { c } { 1 \leq i _ { j } \leq k _ { n } , \\
j = 1 , 2 , \ldots , m \\
i _ { j } \neq i _ { k } \forall k \neq j } }\end{subarray}}^{k-1} *\left(\begin{array}{c}
k \\
s_{1}, \ldots, s_{i_{m}}
\end{array}\right)\left(\prod_{h=1_{s_{i_{h}}} \geq 2}^{L} E\left(\frac{\left\|X_{n i_{j_{h}}}^{\prime}\right\|^{p}}{\left|a_{n i_{j_{h}}}^{-1}\right|^{p}}\right)^{s_{i_{h}}}\right)\right) \\
\left.\cdot\left(\prod_{j=1}^{N} E \frac{\left\|X_{n i_{h_{j}}}^{\prime}\right\|^{p}}{\left|a_{n i_{h_{j}}}^{-1}\right|^{p}}\right)\right\},
\end{gathered}
$$

where $L=$ number of $s_{i} \geq 2, N=$ number of $s_{i}=1$, and $\left\{s_{i_{1}}, \ldots, s_{i_{m}}\right\}=\left\{s_{i_{j_{h}}}, h=1, \ldots, L\right\} \cup$ $\left\{s_{i_{h_{j}}}, s_{i_{h_{j}}}=1, h=1, \ldots, N\right\},\left\{s_{i_{j_{h}}}, h=1, \ldots, L\right\} \cap\left\{s_{i_{h_{j}}}, s_{i_{h_{j}}}=1, h=1, \ldots, N\right\}=\varnothing$.

But

$$
\left(\frac{\|\left. X_{n i_{j}}^{\prime}\right|^{p}}{\left|a_{n i_{j}}^{-1}\right|^{p}}\right)^{s_{i_{j}}} \leq \frac{\|\left. X_{n i_{j}}^{\prime}\right|^{p}}{\left|a_{n i_{j}}^{-1}\right|^{p}}, \quad E \frac{\left\|X_{n i_{j}}^{\prime}\right\|^{p}}{\left|a_{n i_{j}}^{-1}\right|^{p}} \leq \sum_{i=1}^{k_{n}} \frac{E \|\left. X_{n i_{j}}^{\prime}\right|^{p}}{\left|a_{n i_{j}}^{-1}\right|^{p}} \text { for } 1 \leq i_{j} \leq k_{n},
$$

so the right-hand side of (33) can be estimated as follows:

$$
\begin{aligned}
C \sum_{n=1}^{\infty} & \left\{\left(\sum_{i=1}^{k_{n}} E \frac{\left\|X_{n i}^{\prime}\right\|^{p}}{\left|a_{n i}^{-1}\right|^{p}}\right)^{k}+\left(\sum_{i=1}^{k_{n}} E \frac{\left\|X_{n i}^{\prime}\right\|^{p}}{\left|a_{n i}^{-1}\right|^{p}}\right)^{L} \cdot\left(\sum_{i=1}^{k_{n}} E \frac{\left\|X_{n i}^{\prime}\right\|^{p}}{\left|a_{n i}^{-1}\right|^{p}}\right)^{M}\right\} \\
& \leq C \sum_{n=1}^{\infty}\left(\sum_{i=1}^{k_{n}} E \frac{\left\|X_{n i}^{\prime}\right\|^{p}}{\left|a_{n i}^{-1}\right|^{p}}\right)^{k}<\infty .
\end{aligned}
$$

Therefore, assumption (14) of Theorem 4 holds. Moreover, by (31), we get (15) and (16) of Theorem 4 . We also note that if $\mathscr{B}$ is a Banach space of Rademacher type $p, 1<p \leq 2$, we have the following estimation:

$$
P\left[\left\|S_{n}\right\| \geq \varepsilon\right] \leq \varepsilon^{-p} E\left\|S_{n}\right\|^{p} \leq \varepsilon \sum_{i=1}^{k_{n}} E\left\|a_{n i} X_{n i}\right\|^{p} \leq \varepsilon^{-p} \sum_{i=1}^{k_{n}} \frac{E\left(\left\|X_{n i}\right\|^{p}\right)}{\left|a_{n i}\right|^{-p}}=o(1) .
$$

This fact, together with (20), completes the proof.

Now we present the result which gives the sufficient conditions for the equivalence of (17) and (18) in the Chung-Teicher terms (cf. Teicher [13]).

THEOREM 7. Let $\left\{X_{n i}, 1 \leq i \leq k_{n}, n \geq 1\right\}$ be an array of row-wise independent $\mathscr{B}$ valued random variables with $E X_{n i}=0$ for all $1 \leq i \leq k_{n}, n \geq 1$, and for some increasing sequence $\left\{k_{n}, n \geq 1\right\}$ of positive integers. Let $\varphi_{n i}: \mathbb{R} \rightarrow \mathbb{R}_{+}$be positive, even, and 
continuous functions which, for constants $\alpha_{n i} \geq 1,0<\beta_{n i} \leq 2, K_{n i}>0$, and $M_{n i}>0$, $n \geq 1, i \geq 1$, satisfy (12) and

$$
\left|x_{1}\right| \leq\left|x_{2}\right| \Longrightarrow \frac{\left|x_{1}\right|^{\beta_{n i}}}{\varphi_{n i}\left(\left|x_{1}\right|\right)} \leq M_{n i} \frac{\left|x_{2}\right|^{\beta_{n i}}}{\varphi_{n i}\left(\left|x_{2}\right|\right)}
$$

Suppose that for some array $\left\{a_{n i},\left(1 \leq i \leq k_{n}, n \geq 1\right)\right\}$ of nonzero reals,

$$
\begin{aligned}
\sum_{n=1}^{\infty} \sum_{i=2}^{k_{n}} M_{n i} & \frac{E \varphi_{n i}\left(\left\|X_{n i}\right\|\right)}{P \varphi_{n i}\left(a_{n i}^{-1}\right)} \sum_{j=1}^{i-1} M_{n j} \frac{E \varphi_{n j}\left(\left\|X_{n j}\right\|\right)}{\varphi_{n j}\left(a_{n j}^{-1}\right)}<\infty, \\
& \sum_{i=1}^{k_{n}} M_{n i} \frac{E \varphi_{n i}\left(\left\|X_{n i}\right\|\right)}{\varphi_{n i}\left(a_{n i}^{-1}\right)}=o(1), \\
& \sum_{i=1}^{k_{n}} K_{n i} \frac{E \varphi_{n i}\left(\left\|X_{n i}\right\|\right)}{\varphi_{n i}\left(a_{n i}^{-1}\right)}=o(1), \\
& \sum_{n=1}^{\infty} \sum_{i=1}^{k_{n}} P\left[\left\|X_{n i}\right\| \geq c_{n i}\right]<\infty
\end{aligned}
$$

for some array $\left\{c_{n i}, i \geq 1, n \geq 1\right\}$ of positive numbers such that

$$
\begin{aligned}
& \sum_{n=1}^{\infty} \sum_{i=1}^{k_{n}} M_{n i}^{2} \cdot \varphi_{n i}\left(c_{n i}\right) \frac{E \varphi_{n i}\left(\left\|X_{n i}\right\|\right)}{\varphi_{n i}^{2}\left(a_{n i}^{-1}\right)}<\infty, \\
& \sum_{n=1}^{\infty} \sum_{i=1}^{k_{n}} K_{n i}^{2} \cdot \varphi_{n i}\left(c_{n i}\right) \frac{E \varphi_{n i}\left(\left\|X_{n i}\right\|\right)}{\varphi_{n i}^{2}\left(a_{n i}^{-1}\right)}<\infty .
\end{aligned}
$$

Then (17) is equivalent to (18).

Proof. Let $X_{n i}^{\prime}=X_{n i} I\left[\left\|X_{n i}\right\| \leq\left|a_{n i}^{-1}\right|\right], X_{n i}^{*}=X_{n i}^{\prime}-E X_{n i}^{\prime}, S_{n}^{\prime}=\sum_{i=1}^{k_{n}} a_{n i} X_{n i}^{\prime}$, and $S_{n}^{*}=$ $\sum_{i=1}^{k_{n}} a_{n i} X_{n i}^{*}$. By (20), we state that $\left\{S_{n}, n \geq 1\right\}$ and $\left\{S_{n}^{\prime}, n \geq 1\right\}$ are equivalent.

Moreover, we have by (40)

$$
\begin{aligned}
\left\|\sum_{i=1}^{k_{n}} a_{n i} E X_{n i} I\left[\left\|X_{n i}\right\| \leq\left|a_{n i}^{-1}\right|\right]\right\| & \leq \sum_{i=1}^{k_{n}}\left|a_{n i}\right| \cdot\left\|E X_{n i} I\left[|| X_{n i} \| \leq\left|a_{n i}^{-1}\right|\right]\right\| \\
& =\sum_{i=1}^{k_{n}}\left|a_{n i}\right| \cdot|| E X_{n i} I\left[|| X_{n i} \|>\left|a_{n i}^{-1}\right|\right] \| \\
& \leq \sum_{i=1}^{k_{n}} \frac{E \| X_{n i}|| I\left[|| X_{n i} \|>\left|a_{n i}^{-1}\right|\right]}{\left|a_{n i}^{-1}\right|} \\
& \leq \sum_{i=1}^{k_{n}} \frac{E \| X_{n i}||^{\alpha_{n i}} I\left[|| X_{n i} \|>\left|a_{n i}^{-1}\right|\right]}{\left|a_{n i}^{-1}\right|^{\alpha_{n i}}} \\
& \leq \sum_{i=1}^{k_{n}} K_{n i} \cdot \frac{E \varphi_{n i}\left(\| X_{n i}||\right)}{\varphi_{n i}\left(a_{n i}^{-1}\right)}=o(1) .
\end{aligned}
$$


Now we define

$$
Y_{n i}=E\left(\| S_{n}^{*}|| \mid \mathscr{F}_{n i}\right)-E\left(\| S_{n}^{*}|| \mid \mathscr{F}_{n i-1}\right),
$$

where $\mathscr{F}_{n i}=\sigma\left(X_{n 1}^{*}, X_{n 2}^{*}, \ldots, X_{n i}^{*}\right)$ and $\mathscr{F}_{n 0}=\{\varnothing, \Omega\}$. Then we have

$$
\left\|S_{n}^{*}\right\|-E\left\|S_{n}^{*}\right\|=\sum_{i=1}^{k_{n}} Y_{n i}
$$

and we note that $\left\{Y_{n i}, 1 \leq i \leq k_{n}\right\}$ is a sequence of martingale differences for a fixed $n$.

Now we are going to prove that

$$
E\left\|S_{n}^{*}\right\| \longrightarrow 0, \quad n \rightarrow \infty
$$

First we will show that

$$
\left\|S_{n}^{*}\right\|-E\left\|S_{n}^{*}\right\| \stackrel{P}{\longrightarrow} 0, \quad n \rightarrow \infty .
$$

Using Chebyshev's inequality, Lemma 3, and assumption (39), we get, for any $\varepsilon>0$,

$$
\begin{aligned}
P[\| & \left.\left\|S_{n}^{*}\right\|-E\left\|S_{n}^{*}\right\| \mid>\varepsilon\right] \\
& \leq \varepsilon^{-2} E\left(\left\|S_{n}^{*}\right\|-E\left\|S_{n}^{*}\right\|\right)^{2}=\varepsilon^{-2} E\left(\sum_{i=1}^{k_{n}} Y_{n i}\right)^{2}=\varepsilon^{-2} \sum_{i=1}^{k_{n}} E\left(Y_{n i}^{2}\right) \\
& \leq \varepsilon^{-2} \sum_{i=1}^{k_{n}} E\left(\left\|a_{n i} X_{n i}^{*}\right\|+E\left\|a_{n i} X_{n i}^{*}\right\|\right)^{2} \leq 8 \varepsilon^{-2} \sum_{i=1}^{k_{n}} a_{n i}^{2} E\left\|X_{n i}^{\prime}\right\|^{2} \\
& =8 \varepsilon^{-2} \sum_{i=1}^{k_{n}} E \frac{\left\|X_{n i}^{\prime}\right\|^{\beta_{n i}}}{\left|a_{n i}^{-1}\right|^{\beta_{n i}}} \cdot \frac{\left\|X_{n i}^{\prime}\right\|^{2-\beta_{n i}}}{\left|a_{n i}^{-1}\right|^{2-\beta_{n i}}} \leq 8 \varepsilon^{-2} \sum_{i=1}^{k_{n}} M_{n i} \frac{E \varphi_{n i}\left(\left\|X_{n i}^{\prime}\right\|\right)}{\varphi_{n i}\left(a_{n i}\right)}=o(1) .
\end{aligned}
$$

Thus, we conclude that (48) holds and, together with (17), (20), and (44), gives (47).

Now we want to show that $\left\|S_{n}^{*}\right\| \rightarrow 0$ a.s., as $n \rightarrow \infty$.

By (47) it is enough to prove that

$$
\left\|S_{n}^{*}\right\|-E\left\|S_{n}^{*}\right\| \longrightarrow 0 \quad \text { a.s., } n \longrightarrow \infty \text {. }
$$

Taking into account the identity

$$
\left(\left\|S_{n}^{*}\right\|-E\left\|S_{n}^{*}\right\|\right)^{2}=\sum_{i=1}^{k_{n}} Y_{n i}^{2}+2 \sum_{i=2}^{k_{n}} Y_{n i} \sum_{j=1}^{i-1} Y_{n j}
$$

and using the notation

$$
Z_{n i}=Y_{n i}^{2} I\left[\left\|X_{n i}\right\|<c_{n i}\right]-E\left(Y_{n i}^{2} I\left[\left\|X_{n i}\right\|<c_{n i}\right] \mid \mathscr{F}_{n i-1}\right), \quad 1 \leq i \leq k_{n},
$$


we have, by Chebyshev's inequality, Lemma 3, and assumption (42),

$$
\begin{aligned}
\sum_{n=1}^{\infty} P\left[\left|\sum_{i=1}^{k_{n}} Z_{n i}\right|>\varepsilon\right] & \leq \varepsilon^{-2} \sum_{n=1}^{\infty} E\left(\sum_{i=1}^{k_{n}} Z_{n i}\right)^{2}=\varepsilon^{-2} \sum_{n=1}^{\infty} \sum_{i=1}^{k_{n}} E\left(Z_{n i}^{2}\right) \\
& \leq C \cdot \varepsilon^{-2} \sum_{n=1}^{\infty} \sum_{i=1}^{k_{n}} E\left(Y_{n i}^{4} I\left[\left\|X_{n i}\right\|<c_{n i}\right]\right) \\
& \leq C \cdot \varepsilon^{-2} \sum_{n=1}^{\infty} \sum_{i=1}^{k_{n}} E\left\|a_{n i} X_{n i}^{\prime}\right\|^{4} I\left[\left\|X_{n i}\right\|<c_{n i}\right] \\
& \leq C \cdot \varepsilon^{-2} \sum_{n=1}^{\infty} \sum_{i=1}^{k_{n}} E\left(\frac{\left\|X_{n i}^{\prime}\right\|^{2 \beta_{n i}}}{\left|a_{n i}^{-1}\right|^{2 \beta_{n i}}} \cdot \frac{\left\|X_{n i}^{\prime}\right\|^{4-2 \beta_{n i}}}{\left.\left|a_{n i}^{-1}\right|^{4-2 \beta_{n i}} I\left[\left\|X_{n i}\right\|<c_{n i}\right]\right)}\right. \\
& \leq C \cdot \varepsilon^{-2} \sum_{n=1}^{\infty} \sum_{i=1}^{k_{n}} M_{n i} \cdot \varphi_{n i}\left(c_{n i}\right) \cdot \frac{E \varphi_{n i}\left(\left\|X_{n i}\right\|\right)}{\varphi_{n i}^{2}\left(a_{n i}^{-1}\right)}<\infty .
\end{aligned}
$$

Hence, by the Borel-Cantelli Lemma, we obtain

$$
\sum_{i=1}^{k_{n}} Y_{n i}^{2} I\left[\left\|X_{n i}\right\|<c_{n i}\right]-\sum_{i=1}^{k_{n}} E\left(Y_{n i}^{2} I\left[\left\|X_{n i}\right\|<c_{n i}\right] \mid \mathscr{F}_{n i-1}\right) \longrightarrow 0 \quad \text { a.s., } n \longrightarrow \infty \text {. }
$$

Using Lemma 3, we can note by assumption (39) that

$$
\begin{aligned}
& \sum_{i=1}^{k_{n}} E\left(Y_{n i}^{2} I\left[\left\|X_{n i}\right\|<c_{n i}\right] \mid \mathscr{F}_{n i-1}\right) \\
& \quad \leq 8 \sum_{i=1}^{k_{n}} E\left(\left\|a_{n i} X_{n i}^{\prime}\right\|^{2}\right) \leq \sum_{i=1}^{k_{n}} E\left(\frac{\left\|X_{n i}^{\prime}\right\|^{\beta_{n i}}}{\left|a_{n i}^{-1}\right|^{\beta_{n i}}} \cdot \frac{\left\|X_{n i}^{\prime}\right\|^{2-\beta_{n i}}}{\left|a_{n i}^{-1}\right|^{2-\beta_{n i}}}\right) \\
& \quad \leq \sum_{i=1}^{k_{n}} M_{n i} \cdot \frac{E \varphi_{n i}\left(\| X_{n i}||\right)}{\varphi_{n i}\left(a_{n i}^{-1}\right)}=o(1),
\end{aligned}
$$

which, together with (54), allows us to state that

$$
\sum_{i=1}^{k_{n}} Y_{n i}^{2} I\left[\left\|X_{n i}\right\|<c_{n i}\right] \longrightarrow 0 \quad \text { a.s., } n \longrightarrow \infty \text {. }
$$

To prove that

$$
\sum_{i=1}^{k_{n}} Y_{n i}^{2} \longrightarrow 0 \quad \text { a.s., } n \longrightarrow \infty,
$$

we only need to show that

$$
\sum_{i=1}^{k_{n}} Y_{n i}^{2} I\left[\left\|X_{n i}\right\| \geq c_{n i}\right] \longrightarrow 0 \quad \text { a.s., } n \longrightarrow \infty
$$


Indeed, by (41) and Lemma 3, we have

$$
\begin{aligned}
\sum_{n=1}^{\infty} P & {\left[\left|\sum_{i=1}^{k_{n}} Y_{n i}^{2} I\left[\| X_{n i}|| \geq c_{n i}\right]\right| \geq \varepsilon\right] } \\
& \leq \varepsilon^{-1} \sum_{n=1}^{\infty} E\left|\sum_{i=1}^{k_{n}} Y_{n i}^{2} I\left[\left\|X_{n i}\right\| \geq c_{n i}\right]\right| \leq C \sum_{n=1}^{\infty} \sum_{i=1}^{k_{n}} E\left\|a_{n i} X_{n i}^{\prime}\right\|^{2} I\left[\left\|X_{n i}\right\| \geq c_{n i}\right] \\
& \leq C \sum_{n=1}^{\infty} \sum_{i=1}^{k_{n}} E I\left[\left\|X_{n i}\right\| \geq c_{n i}\right] \leq \varepsilon^{-1} \sum_{n=1}^{\infty} \sum_{i=1}^{k_{n}} P\left[\left\|X_{n i}\right\| \geq c_{n i}\right]<\infty,
\end{aligned}
$$

and, by the Borel-Cantelli Lemma, we get (57). To close this proof, we must show that

$$
\sum_{i=2}^{k_{n}} Y_{n i} \sum_{j=1}^{i-1} Y_{n j} \longrightarrow 0 \quad \text { a.s., } n \longrightarrow \infty .
$$

Using the fact that $\left\{Y_{n i} \sum_{j=1}^{i-1} Y_{n j}, 2 \leq i \leq k_{n}\right\}$ is a sequence of martingale differences for each $n$, we have, by Chebyshev's inequality, Lemma 3, and assumption (38),

$$
\begin{aligned}
\sum_{n=1}^{\infty} P\left[\left|\sum_{i=2}^{k_{n}} Y_{n i} \sum_{j=1}^{i-1} Y_{n j}\right|>\varepsilon\right] \\
\leq \varepsilon^{-2} \sum_{n=1}^{\infty} \sum_{i=2}^{k_{n}} E\left[\left(\left\|a_{n i} X_{n i}^{*}\right\|+E\left\|a_{n i} X_{n i}^{*}\right\|\right)^{2} \cdot\left(\sum_{j=1}^{i-1} Y_{n j}\right)^{2}\right] \\
\leq \varepsilon^{-2} \sum_{n=1}^{\infty} \sum_{i=2}^{k_{n}} E\left(\left\|a_{n i} X_{n i}^{*}\right\|+E\left\|a_{n i} X_{n i}^{*}\right\|\right)^{2} \cdot \sum_{j=1}^{i-1} E\left(Y_{n j}^{2}\right) \\
\leq C \sum_{n=1}^{\infty} \sum_{i=2}^{k_{n}} E\left\|a_{n i} X_{n i}^{\prime}\right\|^{2} \sum_{j=1}^{i-1} E\left\|a_{n j} X_{n j}^{\prime}\right\|^{2} C \sum_{n=1}^{\infty} \sum_{i=2}^{k_{n}} M_{n i} \frac{E \varphi_{n i}\left(\left\|X_{n i}\right\|\right)}{\varphi_{n i}\left(a_{n i}^{-1}\right)} \\
\quad \times \sum_{j=1}^{i-1} M_{n j} \frac{E \varphi_{n j}\left(\left\|X_{n j}\right\|\right)}{\varphi_{n j}\left(a_{n j}^{-1}\right)}<\infty,
\end{aligned}
$$

which, together with the Borel-Cantelli Lemma, implies (60).

Thus, we have proved that

$$
\sum_{i=1}^{k_{n}} a_{n i}\left(X_{n i}^{\prime}-E X_{n i}^{\prime}\right) \longrightarrow 0 \quad \text { a.s., } n \longrightarrow \infty .
$$

But $\left\{\sum_{i=1}^{k_{n}} a_{n i} X_{n i}^{\prime}, n \geq 1\right\}$ and $\left\{\sum_{i=1}^{k_{n}} a_{n i} X_{n i}, n \geq 1\right\}$ are equivalent and (44) holds, so we get (18).

Now we consider an array $\left\{X_{n i}, i \geq 1, n \geq 1\right\}$ of independent random elements which are stochastically dominated by a random element $X$ in the sense of (10).

COROllary 8. Let $\left\{X_{n i}, 1 \leq i \leq k_{n}, n \geq 1\right\}$ be an array of row-wise independent $\mathscr{B}$-valued random variables with $E X_{n i}=0$ for all $1 \leq i \leq k_{n}, n \geq 1$, and some increasing 
sequence $\left\{k_{n}, n \geq 1\right\}$ of positive integers stochastically dominated by a random element $X$ in the sense of (10). Let $E\|X\|^{p}<\infty, 1<p \leq 2$. Assume that $\left\{a_{n i}, 1 \leq i \leq k_{n}, n \geq 1\right\}$ is an array of nonzero reals.

Suppose that for some increasing sequence $\left\{k_{n}, n \geq 1\right\}$ of positive integers,

$$
\begin{gathered}
\sum_{n=1}^{\infty} \sum_{i=2}^{k_{n}}\left|a_{n i}\right|^{p} \sum_{j=1}^{i-1}\left|a_{n j}\right|^{p}<\infty, \\
\sum_{i=1}^{k_{n}}\left|a_{n i}\right|^{p}=o(1), \\
\sum_{n=1}^{\infty} \sum_{i=1}^{k_{n}} P\left[\|X\| \geq c_{n i}\right]<\infty,
\end{gathered}
$$

for some array $\left\{c_{n i},(1 \leq i \leq 1, n \geq 1)\right\}$ of positive numbers such that

$$
\sum_{n=1}^{\infty} \sum_{i=1}^{k_{n}} c_{n i}^{p} \cdot\left|a_{n i}\right|^{2 p}<\infty
$$

Then (17) is equivalent to (18).

Proof. Put $\varphi_{n i}(x)=|x|^{p}, 1<p \leq 2$, for all $1 \leq i \leq k_{n}, n \geq 1$. Then there exist $\alpha$ and $\beta$ such that $1 \leq \alpha<p<\beta \leq 2$, for which (12) and (37) hold with $\alpha_{n i}=\alpha, \beta_{n i}=\beta$, and $M_{n i}=K_{n i}=1, i \geq 1, n \geq 1$. Moreover, (10) and $E\|X\|^{p}<\infty$ imply $E\left\|X_{n i}\right\|^{p}<\infty$ for all $1 \leq i \leq k_{n}$ and $n \geq 1$. Therefore, we see that assumptions (38)-(43) of Theorem 7 are fulfilled ((38) is fulfilled by (63), (39) and (40) by (64), (41) by (65), and (42)-(43) by (66)).

COROLlaRY 9. Let $\left\{X_{n i},(1 \leq i \leq n, n \geq 1)\right\}$ be an array of row-wise independent $\Re$-valued random variables with $E X_{n i}=0$ for $1 \leq i \leq n, n \geq 1$, stochastically dominated by a random element $X$ in the sense of (10). Assume that $\left\{a_{n i}, i \geq 1, n \geq 1\right\}$ is a Toeplitz array such that $a_{n i} \neq 0, i \geq 1, n \geq 1$, and for some $\gamma$ such that $\gamma p>2,1<p \leq 2$,

$$
\sup _{i \geq 1}\left|a_{n i}\right|=O\left(n^{-\gamma}\right)
$$

If $E\|X\|^{p}<\infty$, then (17) is equivalent to (18).

Proof. To prove this result, it is enough to show that under assumption (67), conditions (14), (15), and (16) of Theorem 4 are fulfilled.

Indeed, we see that for $\psi_{n i}(x)=\varphi_{n i}(x)=|x|^{p}$ and $M_{n i}=K_{n i}=1, i \geq 1, n \geq 1, k=1$, and $k_{n}=n, n \geq 1$, we have

$$
\sum_{n=1}^{\infty} E\left(\sum_{i=1}^{n}\left|a_{n i}\right|^{p} \|\left. X_{n i}\right|^{p}\right) \leq C \sum_{n=1}^{\infty}\left(\sup _{i \geq 1}\left|a_{n i}\right|\right)^{p} \sum_{i=1}^{n} E\|X\|^{p} \leq C \sum_{n=1}^{\infty} \frac{1}{n^{\gamma^{p}-1}}<\infty .
$$

So, condition (14) is fulfilled. 
For $c_{n i}=D / a_{n i}, i \geq 1, n \geq 1$, we get (15) and (16):

$$
\begin{aligned}
& \sum_{n=1}^{\infty} \sum_{i=1}^{n} P\left[\left\|X_{n i}\right\| \geq \frac{D}{a_{n i}}\right] \\
& \leq \sum_{n=1}^{\infty} \sum_{i=1}^{n} P\left[D\|X\| \geq \frac{D}{a_{n i}}\right] \leq C \sum_{n=1}^{\infty} \sum_{i=1}^{n}\left|a_{n i}\right|^{p} E\|X\|^{p} \leq C \sum_{n=1}^{\infty} \frac{1}{n^{\gamma p-1}}<\infty, \\
& \sum_{n=1}^{\infty} \sum_{i=1}^{n}\left|\frac{D}{a_{n i}}\right|^{p}\left|a_{n i}\right|^{2 p} E\left\|X X_{n i}\right\|^{p} \leq C \sum_{n=1}^{\infty} \sum_{i=1}^{n}\left|a_{n i}\right|^{p} E\|X\|^{p} \leq C \sum_{n=1}^{\infty} \frac{1}{n^{\gamma p-1}}<\infty .
\end{aligned}
$$

Similarly, we can prove the next corollary.

COROLlary 10. Let $\left\{X_{n i},(1 \leq i \leq n, n \geq 1)\right\}$ be an array of row-wise independent B-valued random variables with $E X_{n i}=0$ for $1 \leq i \leq k_{n}, n \geq 1$, and some increasing sequence $\left\{k_{n}, n \geq 1\right\}$ of positive integers stochastically dominated by a random element $X$ in the sense of (10). Assume that $\left\{a_{n i}, i \geq 1, n \geq 1\right\}$ is a Toeplitz array such that $a_{n i} \neq 0, i \geq 1, n \geq 1$, and for some $\gamma$ such that $\gamma q>2,0<q \leq 2$,

$$
\sum_{i=1}^{k_{n}}\left|a_{n i}\right|^{q}=O\left(n^{-\gamma}\right)
$$

If $E\|X\|^{q}<\infty$, then (17) is equivalent to (18).

Using the fact that $\mathscr{B}$ is a Banach space of Rademacher type $p$, we see that (67) implies (17) under $E\|X\|^{p}<\infty$. Indeed, we see that

$$
P\left[\left\|\sum_{i=1}^{n} a_{n i} X_{n i}\right\| \geq \varepsilon\right] \leq \varepsilon^{-p} \sum_{i=1}^{n} E\left\|a_{n i} X_{n i}\right\|^{p} \leq C \sum_{i=1}^{n}\left|a_{n i}\right|^{p} E\|X\|^{p} \leq \frac{C}{n^{\gamma p-1}}=o(1) .
$$

So, we can formulate the next result.

Corollary 11. Let $\left\{X_{n i}, 1 \leq i \leq n, n \geq 1\right\}$ be an array of row-wise independent random elements taking values in a Banach space of Rademacher type $p, 1<p \leq 2$, with $E X_{n i}=0$ for $i \geq 1, n \geq 1$, stochastically dominated by a random element $X$ in the sense of (10) and $E\|X\|^{p}<\infty$. Assume that $\left\{a_{n i}, i \geq 1, n \geq 1\right\}$ is a Toeplitz array. If for some $\gamma$ satisfying $\gamma p>2$ and $a_{n i} \neq 0, i \geq 1, n \geq 1$, (67) holds, then (18) is fulfilled.

EXAMPLE 12. Let $\left\{X_{n}, n \geq 1\right\}$ be a sequence of independent random variables which are stochastically dominated by a random variable $X$ such that $E X=0$ and $E|X|^{\gamma}<\infty$ for some $\gamma, 1 \leq \alpha<\gamma<\beta \leq 2$. Set $X_{n i}=X_{i}^{i} / n^{(1+\varepsilon) i / \gamma}$ and $a_{n i}=1 / n^{i / \gamma}$, for $1 \leq i \leq n$, $n \geq 1$.

We will verify that conditions (14), (15), and (16) of Theorem 4 hold with $k_{n}=n, n \geq 1$, $c_{n i}=n^{i / \gamma}$, and $\varphi_{n i}(x)=\psi_{n i}(x)=|x|^{\gamma / i}, 1 \leq i \leq n, n \geq 1, k=1$.

We note that the functions $\varphi_{n i}$ and $\psi_{n i}, 1 \leq i \leq n, n \geq 1$, satisfy conditions (12) and (13) with $\alpha_{n i}=\alpha / i, \beta_{n i}=\beta / i$, and $M_{n i}=K_{n i}=1$. 
To verify (14), note that

$$
\sum_{n=1}^{\infty} E\left(\sum_{i=1}^{n} M_{n i} \frac{\psi_{n i}\left(\left|X_{n i}\right|\right)}{\psi_{n i}\left(a_{n i}^{-1}\right)}\right) \leq C \sum_{n=1}^{\infty} \sum_{i=1}^{n} \frac{E\left|X_{n i}\right|^{\gamma}}{n} \leq C \sum_{n=1}^{\infty} \sum_{i=1}^{n} \frac{E\left|X_{i}\right|^{\gamma}}{n^{1+\varepsilon} \cdot n} \leq C \sum_{n=1}^{\infty} \frac{1}{n^{1+\varepsilon}}<\infty,
$$

and to verify (15), note that

$$
\begin{aligned}
& \sum_{n=1}^{\infty} \sum_{i=1}^{n} P\left[\left|X_{n i}\right|>n^{i / \gamma}\right] \\
& \quad \leq \sum_{n=1}^{\infty} \sum_{i=1}^{n} P\left[\frac{\left|X_{i}\right|^{i}}{n^{(1+\varepsilon) i / \gamma}}>n^{i / \gamma}\right] \leq \sum_{n=1}^{\infty} \sum_{i=1}^{n} P\left[\left|X_{i}\right|^{i}>n^{(2+\varepsilon) i / \gamma}\right] \leq C \sum_{n=1}^{\infty} \frac{1}{n^{1+\varepsilon}}<\infty
\end{aligned}
$$

Moreover, we see that

$$
\begin{aligned}
& \sum_{n=1}^{\infty} \sum_{i=1}^{n} K_{n i}^{2} \cdot \varphi_{n i}\left(c_{n i}\right) \frac{E \varphi_{n i}\left(\left|X_{n i}\right|\right)}{\varphi_{n i}^{2}\left(a_{n i}^{-1}\right)} \\
& \quad \leq \sum_{n=1}^{\infty} \sum_{i=1}^{n} \frac{n E\left|X_{n i}\right|^{\gamma / i}}{n^{2}} \leq \sum_{n=1}^{\infty} \sum_{i=1}^{n} \frac{E\left|X_{i}\right|^{\gamma}}{n^{2+\varepsilon}} \leq \sum_{n=1}^{\infty} \frac{1}{n^{1+\varepsilon}}<\infty .
\end{aligned}
$$

To complete the proof, we note that the real number space is of Rademacher type $p$ with $p=2$, so by the estimations (36), (72), and (73), we have (17). Thus, by Theorem 4, we have

$$
\sum_{i=1}^{\infty} a_{n i} X_{n i}=\sum_{i=1}^{n} n^{-(2+\varepsilon) i / \gamma} X_{i}^{i} \longrightarrow 0, \quad \text { a.s., } n \longrightarrow \infty .
$$

ACKNOWLEDGMENT. The author is very grateful to the referee for the suggestions that allowed her to improve this paper.

\section{REFERENCES}

[1] A. Adler, A. Rosalsky, and R. L. Taylor, Some strong laws of large numbers for sums of random elements, Bull. Inst. Math. Acad. Sinica 20 (1992), no. 4, 335-357.

[2] E. Berger, Majorization, exponential inequalities and almost sure behavior of vector-valued random variables, Ann. Probab. 19 (1991), no. 3, 1206-1226.

[3] A. Bozorgnia, R. F. Patterson, and R. L. Taylor, Chung type strong laws for arrays of random elements and bootstrapping, Stochastic Anal. Appl. 15 (1997), no. 5, 651-669.

[4] A. de Acosta, Inequalities for B-valued random vectors with applications to the strong law of large numbers, Ann. Probab. 9 (1981), no. 1, 157-161.

[5] N. Etemadi, Tail probabilities for sums of independent Banach space valued random variables, Sankhyā Ser. A 47 (1985), no. 2, 209-214.

[6] J. Hoffmann-Jørgensen and G. Pisier, The law of large numbers and the central limit theorem in Banach spaces, Ann. Probab. 4 (1976), no. 4, 587-599.

[7] T.-C. Hu and R. L. Taylor, On the strong law for arrays and for the bootstrap mean and variance, Int. J. Math. Math. Sci. 20 (1997), no. 2, 375-382.

[8] A. Kuczmaszewska and D. Szynal, On the strong law of large numbers in Banach space of type p, Ann. Univ. Mariae Curie-Skłodowska Sect. A 45 (1991), 71-81. 
[9] _ On conditions for the strong law of large numbers in general Banach spaces, Int. J. Math. Math. Sci. 24 (2000), no. 1, 29-42.

[10] J. Kuelbs and J. Zinn, Some stability results for vector valued random variables, Ann. Probab. 7 (1979), no. 1, 75-84.

[11] T. Mikosch and R. Norvaiša, On almost sure behavior of sums of independent Banach space valued random variables, Math. Nachr. 125 (1986), 217-231.

[12] _ Strong laws of large numbers for fields of Banach space valued random variables, Probab. Theory Related Fields 74 (1987), no. 2, 241-253.

[13] H. Teicher, Some new conditions for the strong law, Proc. Nat. Acad. Sci. U.S.A. 59 (1968), 705-707.

[14] X. C. Wang, M. B. Rao, and X. Y. Yang, Convergence rates on strong laws of large numbers for arrays of rowwise independent elements, Stochastic Anal. Appl. 11 (1993), no. 1, 115-132.

[15] W. A. Woyczyński, Random series and laws of large numbers in some Banach spaces, Teor. Verojatnost. i Primenen. 18 (1973), 361-367.

[16] V. V. Yurinskii, Exponential bounds for large deviations, Theor. Probability Appl. 19 (1974), 154-155.

Anna Kuczmaszewska: Department of Applied Mathematics, Lublin University of Technology, Nadbystrzycka 38D, 20-618 Lublin, Poland

E-mail address: a.kuczmaszewska@po11ub.p1 


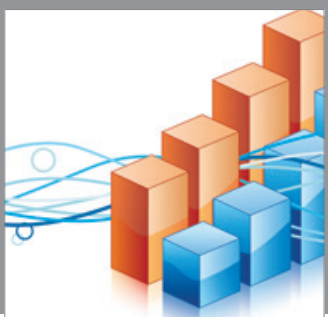

Advances in

Operations Research

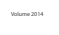

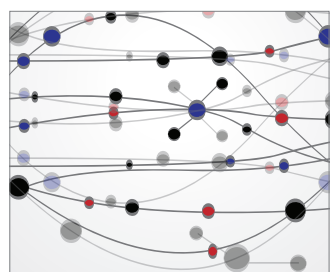

\section{The Scientific} World Journal
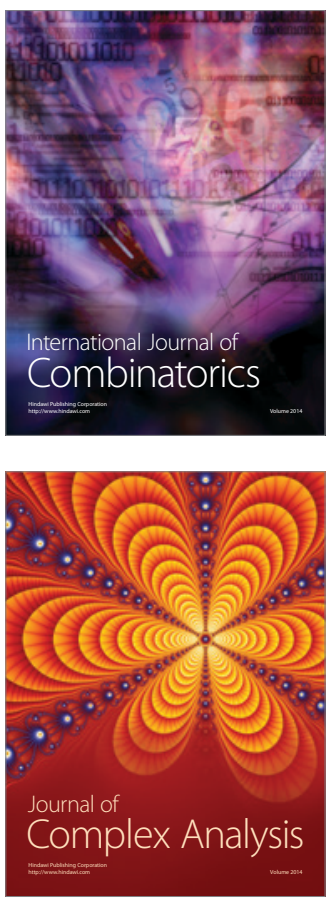

International Journal of

Mathematics and

Mathematical

Sciences
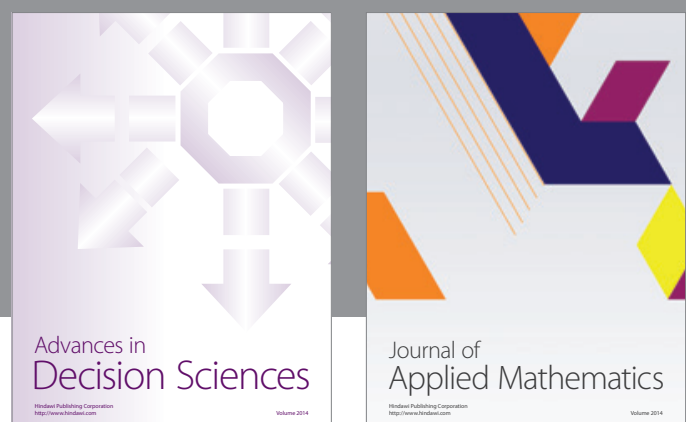

Journal of

Applied Mathematics
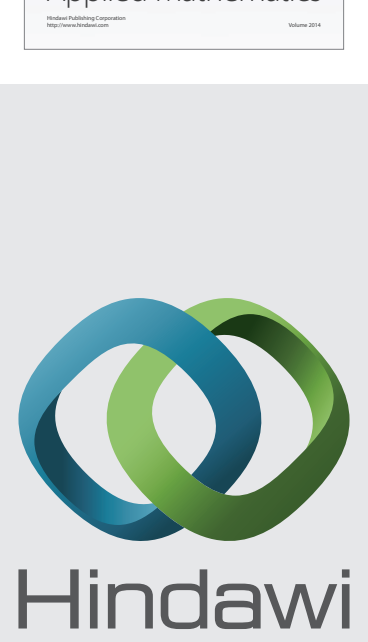

Submit your manuscripts at http://www.hindawi.com
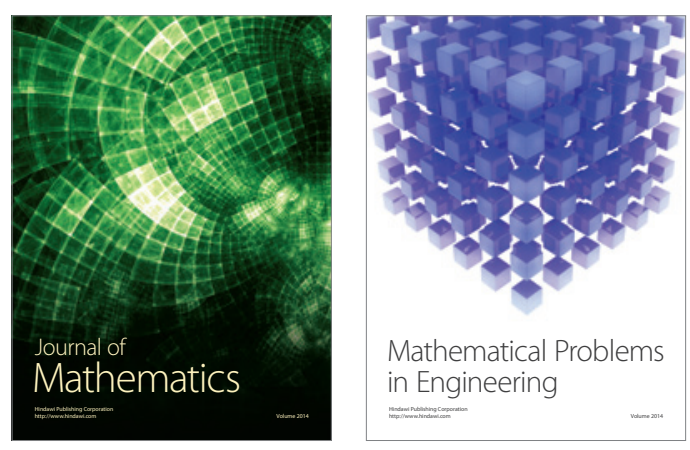

Mathematical Problems in Engineering
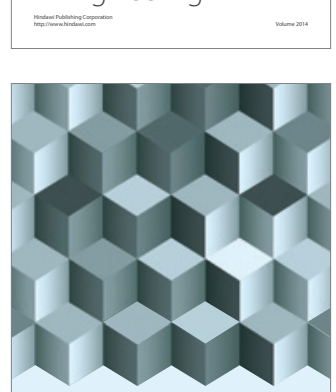

Journal of

Function Spaces
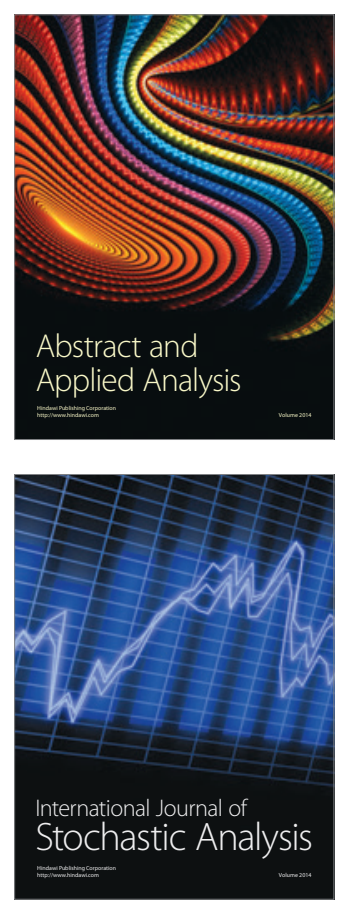

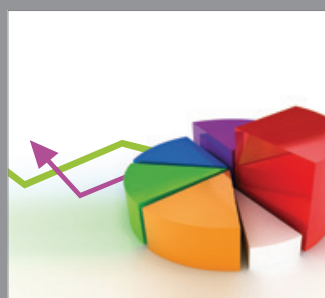

ournal of

Probability and Statistics

Promensencen
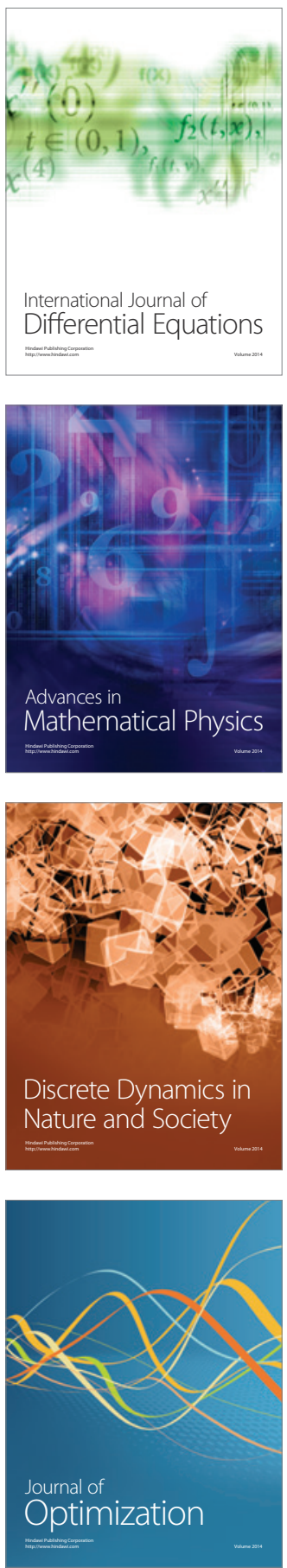